\title{
Signal transduction mechanism of exosomes in diabetic complications (Review)
}

\author{
XUETING LI $^{1}$, SHUO SHI ${ }^{2}$, DEHUAI JING ${ }^{3}$, XINJIAN $\mathrm{LI}^{4}$, \\ BIN ZHANG $^{1,2}$ and QINGLI BIE ${ }^{1,2}$ \\ ${ }^{1}$ Department of Clinical Medicine, Jining Medical University; \\ Departments of ${ }^{2}$ Laboratory Medicine, ${ }^{3}$ Digestive Endoscopy and ${ }^{4}$ Nephrology, \\ Affiliated Hospital of Jining Medical University, Jining, Shandong 272000, P.R. China
}

Received August 13, 2021; Accepted November 24, 2021

DOI: $10.3892 /$ etm.2021.11078

\begin{abstract}
Exosomes are vesicles with a particle size of 30-120 nm that are secreted by cells through exocytosis. The composition of an exosome includes a lipid bilayer and its internal package of biological molecules, such as proteins, ribonucleotides and deoxyribonucleotides. Diabetes is a chronic and refractory disease. The complications induced by high blood glucose have become a major problem in global public health and the pathogenesis of diabetic complications remains to be fully elucidated. In recent years, it has been gradually recognized that exosomes from different cell sources and their related molecules, particularly exosomal proteins and microRNAs, have an important role in the pathogenesis of diabetic complications, allowing for the exploration of the pathogenesis of diabetic complications from a molecular perspective. The present review summarizes the latest studies on exosomes from different cell sources in the pathogenesis of diabetic complications, which may provide novel targets for the prevention and treatment of diabetic complications.
\end{abstract}

\section{Contents}

1. Introduction

2. Development of exosomes

3. Exosomes and diabetes

4. Conclusion

Correspondence to: Professor Bin Zhang or Dr Qingli Bie, Department of Laboratory Medicine, Affiliated Hospital of Jining Medical University, 89 Guhuai Road, Jining, Shandong 272000, P.R. China

E-mail: zhb861109@163.com

E-mail: xiaobie890101@163.com

Key words: molecule, exosomes, pathogenesis, diabetic, complications

\section{Introduction}

China ranks first in the world in terms of incidence of diabetes and the age of patients diagnosed with diabetes exhibits a decreasing trend (1). Diabetes is a metabolic disorder characterized by hyperglycemia caused by insufficient insulin secretion, insulin resistance or both (2). In recent years, exosomes have been demonstrated not only to be biomarkers of diabetic complications but also to participate in the etiology of diabetes $(3,4)$. Therefore, exosomes are helpful for identifying diabetic complications at an early stage and may be used to diagnose and treat diabetes at the molecular level. The present article reviews the role of exosomes in the pathogenesis of diabetic complications and discusses the prospects of using exosomes in the diagnosis and treatment of diabetic complications.

\section{Development of exosomes}

Exosomes are cell vesicles with lipid bilayer membranes with a diameter measuring 30-120 nm that contain specific proteins, related metabolites, lipids and nucleic acids (5). Exosomes not only transmit information between cells in autocrine, paracrine and telecrine states but also correspond to cells (6). Exosomes may be released by most cells in the body and are present in most bodily fluids, such as plasma, urine, saliva, cerebrospinal fluid and breast milk, bronchoalveolar lavage fluid and epididymal fluid (7). Exosomes may be separated by various methods, including ultrahigh-speed centrifugation, filtration, precipitation and immune enrichment (8). They have complex components, including DNA fragments, circular RNA, mRNA, microRNA (miRNA/miR), functional proteins, transcription factors and other substances. Internal vesicles are secreted into the extracellular environment through the fusion of vesicle endosomes and the plasma membrane and then bind with receptor cells to exchange information between cells by releasing substances into receptor cells (9). In addition, exosomes have been demonstrated to have an important role in tumor diagnosis, migration and growth, as well as tissue damage repair, immune antigen presentation and neurodegenerative diseases (10). 


\section{Exosomes and diabetes}

Diabetes is a group of metabolic diseases characterized by hyperglycemia. The etiology, pathogenesis and complexity of diabetes have remained to be fully clarified (11). Diabetes may be divided into two categories according to its pathogenesis as follows: Type 1 diabetes mellitus (T1DM), in which there is a lack of insulin due to the destruction of islet $\beta$ cells, and type 2 diabetes mellitus (T2DM), in which insulin resistance is accompanied by insulin deficiency or impaired insulin secretion (12). Persistent hyperglycemia may induce diabetic complications by regulating the epigenetics of target cells (13). Exosomes have important roles in the pathology of T1DM and T2DM, including the destruction of pancreatic tissue by mediating immune reactions and inducing adipocytes to reduce insulin resistance (14). In recent years, studies have indicated that exosomes carrying miRNAs and long noncoding RNAs have important roles in the development of diabetes by regulating metabolic signals, insulin signaling and the interaction between inflammatory pancreatic cells (15). In addition, miRNAs carried by exosomes may be used as biomarkers in diabetes and diabetic complications (16), which may help to identify diabetic complications at an early stage, thereby allowing patients to receive effective treatment in time.

\section{Role of exosomes in TIDM}

In the vast majority of cases of T1DM, the disease is an autoimmune condition (17). Studies have indicated that the damage to islet $\beta$ cells may be related to exosomes from specific sources. Exosomes from rats and humans contain $\beta$-cell autoantigens, such as $65 \mathrm{kDa}$ glutamic acid decarboxylase, islet antigen-2 and proinsulin, which activate dendritic cells, leading to an increase in cytokine levels and ultimately inducing apoptosis and endoplasmic reticulum stress to destroy islet cells. Exosomes also promote the release of exosomes, forming a vicious cycle that participates in the occurrence and development of T1DM (18). In addition, mesenchymal stem cells (MSCs) derived from pancreatic islets in nonobese diabetic mice were observed to release immunomodulatory exosomes and promote the $\mathrm{T}$ cell-mediated destruction of pancreatic islets (19). This evidence suggests that exosomes promote the development of T1DM. By contrast, in clinical patients with T1DM, upregulation of miR-21-5p and miR-25-3p, as well as downregulation of miR-16-5p, miR-302d-3p, miR-378a, miR-570-3p, miR-574-5p and miR-579, were detected in serum-derived exosomes (Sexos) (20). These exosomes may be used as potential biomarkers of T1DM, which is helpful for its early diagnosis. Regarding treatment in rats, exosomes derived from MSCs (MSC-Exos) contained miR-106b-5p and miR-222-3p, mediated protein kinase B (PKB/Akt and MAPK signaling pathways) and promoted islet $\beta$-cell proliferation, and these exosomes are expected to reverse the occurrence of T1DM in the future (21).

\section{Role of exosomes in T2DM}

T2DM is closely related to obesity (22). Adipose tissues release various mediators, including free fatty acids, tumor necrosis factor, leptin and resistin, leading to systemic insulin resistance (23). Studies in rats have indicated that the miR-34a carried by exosomes derived from adipocytes inhibits the polarization of M2 macrophages and promotes fat inflammation induced by obesity, thereby aggravating the progression of T2DM (24). In addition, MSC-Exos contain miR-122, miR-192, miR-27a-3p and miR-27b-3p, which target peroxisome proliferator-activated receptors $\gamma$ in the adipose tissue of rats, causing adipose tissue degeneration and infiltration of inflammatory factors, ultimately participating in the occurrence and development of T2DM (25). Regarding treatment, studies have indicated that the replication of pancreatic $\beta$-cells declines sharply with age (26), leading to an increased risk of T2DM. Exosomes derived from human umbilical cord mesenchymal stem cells (HucMSC-Exos) carrying miR-16 restored the phosphorylation of insulin receptor substrate 1 and PKB (tyrosine site) in T2DM and promoted the expression of glucose transporter 4 (GLUT4) in muscle and membrane translocation to reverse peripheral insulin resistance (27).

\section{Role of exosomes in diabetic complications}

Diabetic complications include microangiopathy, atherosclerotic cardiovascular disease, neurological complications and diabetic foot ulcers (28). Microvascular disease is a specific complication of diabetes and its typical changes are basement membrane thickening and microcirculation disease. Microvascular disease may affect all tissues and organs in the whole body, and diabetic nephropathy and retinopathy are the most common (29). Experiments in rats have indicated that MSC-Exos reduce oxidative stress by inhibiting C-C motif chemokine receptor 2 and reduce the damage from the inflammatory response to its microvascular endothelium (30); therefore, exosomes may promote angiogenesis and enhance the repair of vascular endothelium damaged by oxidative stress. Exosomes derived from adipose mesenchymal stem cells (AMSC-Exos) are rich in miR-125a, which may inhibit the expression of the angiogenesis inhibitor delta-like 4 protein and promote angiogenesis in rats (31). It has been indicated that the complement system has an important role in the development of vascular damage (32). Furthermore, Huang et al (33) reported that a lack of $\operatorname{IgG}$ in exosomes results in a reduction in vascular damage in diabetic mice. Another study suggested that miR-21 carried by exosomes derived from glioma stem cells upregulates vascular endothelial growth factor expression, which promotes the angiogenic ability of endothelial cells (30). Therefore, exosomes may be a promising drug candidate for treating microvascular damage caused by high glucose.

Diabetic nephropathy $(D N)$. DN is a complex and common chronic kidney disease, and it is one of the primary causes of end-stage renal disease (34). The clinical features of DN are persistent albuminuria and the thickening of the glomerular basement membrane. DN causes an accumulation of the extracellular matrix and induces autophagy, causing podocyte damage and progressive renal insufficiency (35). The traditional method for diagnosing DN is to detect protein in urine; however, as the deterioration of renal function usually occurs earlier than proteinuria, DN cannot be prevented at an early stage (36). It has been reported that podocytes induced by high glucose secrete exosomes carrying early flowering 3 (Elf3), which may only be detected in the urine of patients with DN, suggesting that measuring the level of Elf3 protein in exosomes 


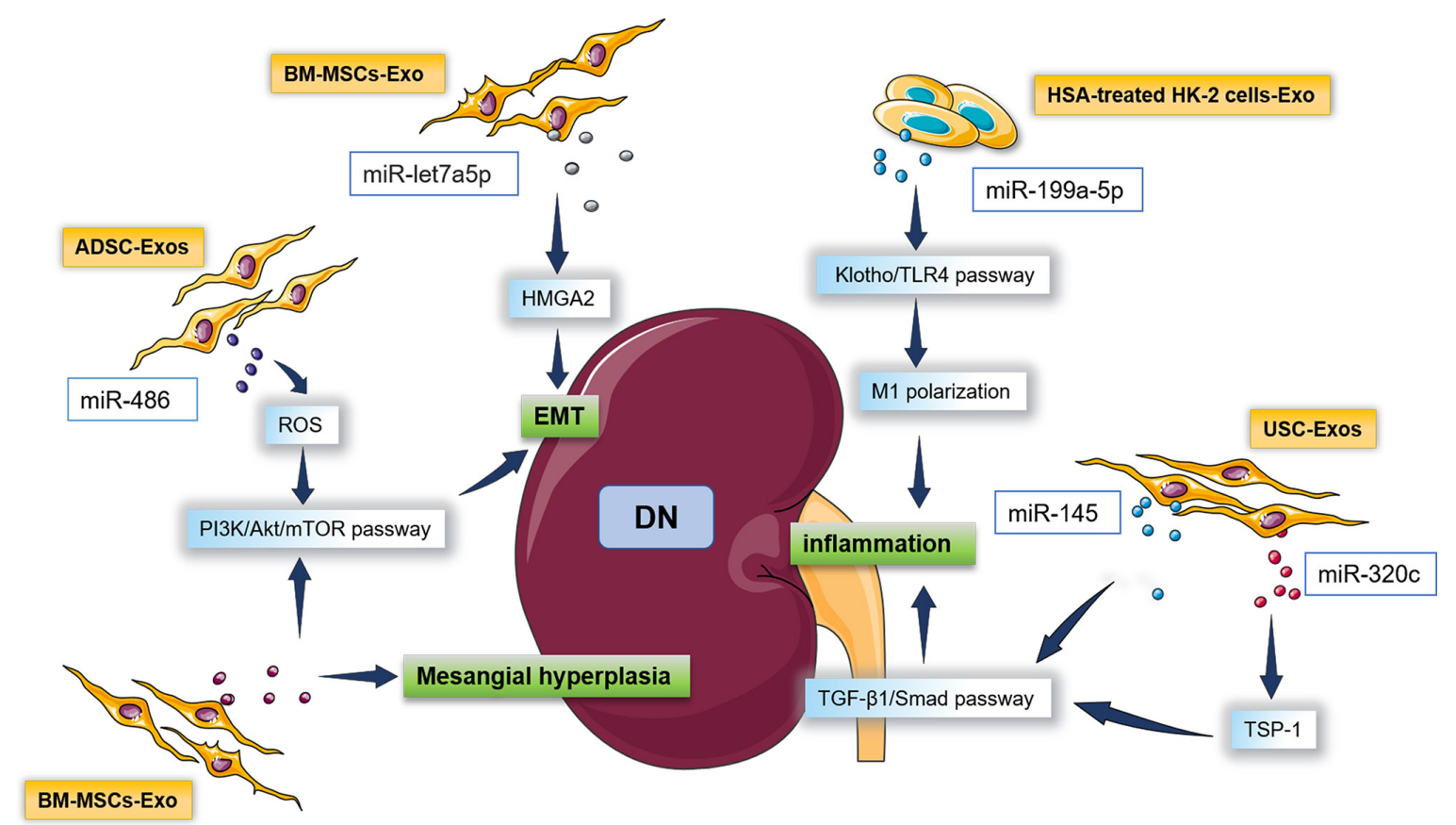

Figure 1. Exosomes are involved in the pathogenesis of DN. BM-MSCs-Exo carry miR-let7a-5p, which acts on HMGA2 to promote the formation of DN renal tubular epithelial cells and renal fibrosis. miR-145 and miR-320c carried by USC-Exos activate the TGF- $\beta 1 /$ Smad pathway to promote the renal fibrosis process. HSA-treated HK-2 cell-Exos release miR-199a-5p, which targets the Klotho/TLR4 pathway to accelerate the inflammatory reaction. BM-MSCs-Exo induce glomerular hypertrophy by promoting the PI3K/Akt/mTOR pathway. miR-486 carried by ADSC-Exos activates reactive oxygen species targeting the PI3K/Akt/mTOR pathway to accelerate the apoptosis of podocytes. DN, diabetic nephropathy; miR, microRNA; BM-MSCs-Exo, exosomes derived from bone marrow mesenchymal stem cells; ADSC, adipose stem cell; HSA, human serum albumin; TLR, Toll-like receptor; ROS, reactive oxygen species; EMT, epithelial to mesenchymal transition; USC, urine-derived stem cell; M1, M1-type macrophage; TSP-1, thrombospondin-1.

derived from the human urine stem cells (USC-Exos) of patients with DN may be used as an early, non-invasive marker of cell damage in podocytes (37). The expression of Wilms tumor protein 1 (WT1) in USC-Exos of patients increases with the decline in the patient's renal function, suggesting that WT1 may be used as a monitoring indicator of early renal function in DN (38). The expression of miR-19b-3p in the USC-Exos of patients with DN is significantly increased and it is positively correlated with the severity of renal interstitial inflammatory reactions in patients with DN, suggesting that miR-19b-3p can be used as a molecular marker for monitoring the progression of DN (39). Fibrosis is a key step in the pathology of DN (40). Exosomes derived from bone marrow MSCs (BM-MSCs-Exo) carry miR-let7a-5p, which acts on high mobility group AT-hook 2 to promote the transformation of DN renal tubular epithelial cells and renal fibrosis in rats (41). In addition, USC-Exos carry miR-145 and miR-320c, which directly or indirectly activate the TGF- $\beta 1 /$ Smad pathway of renal cells and promote renal fibrosis in rats (42). High glucose and proteinuria synergistically stimulate renal podocytes and renal tubular cells to produce chemokines, stimulate the release of inflammatory factors, increase the synthesis of extracellular matrix and accelerate glomerular sclerosis (43). In addition, exosomes secreted from human serum albumin-treated HK-2 cells released miR-199a-5p, which targeted the Klotho/Toll-like receptor (TLR)4 pathway to induce M1-type macrophage (M1) polarization, accelerating the inflammatory reaction and promoting the progression of DN (44). In clinical patients, molecules derived from BM-MSCs-Exo were observed to induce glomerular hypertrophy by promoting the
$\mathrm{PI} 3 \mathrm{~K} / \mathrm{Akt} / \mathrm{mTOR}$ pathway and participate in the proliferation of mesangial cells and the extracellular matrix (45). Adipose stem cell-derived exosomes (ADSC-Exos) containing miR-486 activate reactive oxygen species (ROS), thereby targeting the $\mathrm{PI} 3 \mathrm{~K} / \mathrm{Akt} / \mathrm{mTOR}$ pathway to accelerate the apoptosis of podocytes, and they were observed to participate in the occurrence of DN in rats (46). Exosomes not only serve as related markers of DN but also participate in the pathophysiological processes of DN. Fig. 1 provides a schematic of the mechanisms of the involvement of exosomes in the pathogenesis of DN.

Diabetic retinopathy $(D R)$. Diabetic retinopathy (DR) is a common complication of diabetes. Long-term chronic hyperglycemia may cause retinal microcirculation disorders, which manifest as vascular degeneration in the early stage and develop into proliferative DR during the late stage, which is accompanied by proliferative neovascularization, leading to vitreous bodies and even retinal detachment (47). In recent years, studies have indicated that exosomes reflect the relationship between cell secretion and the progression of DR (33). Mazzeo et al (48) compared the miRNA expression profiles of plasma extracellular vesicles (including exocytosis) between patients with DR and healthy individuals and observed that the expression of miR-150-5p, miR-21-3p and miR-30b-5p was increased in patients with DR, indicating that exosomes carrying these miRNAs may be used as biomarkers for DR. The pathogenesis of DR is complex and remains to be fully elucidated, but it is primarily related to damage to the vascular endothelium and the progression of vascular inflammation and oxidative stress (49). Fig. 2 


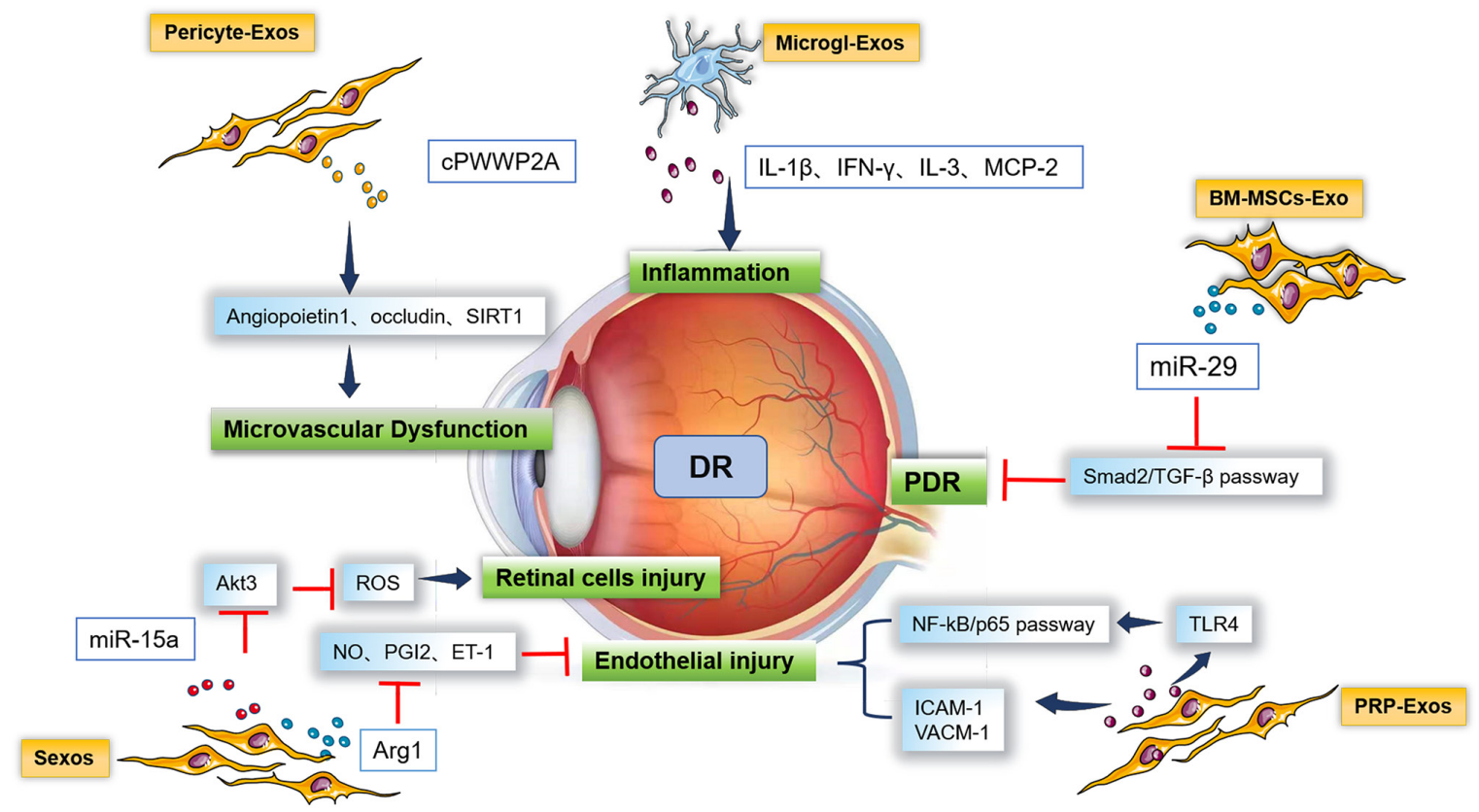

Figure 2. Signal transduction of exosomes in DR. Sexos inhibit NO, ET-1, prostacyclin and PGI2 to promote vascular endothelial damage in DR. miR-15a carried by Sexos inhibits Akt 3 to activate ROS, destroying retinal cells. PRP-Exos target TLR4, activate the NF-kB/p65 pathway and cause damage to retinal endothelial cells. miR-29 carried by BM-MSCs-Exo inhibits the Smad2/TGF- $\beta$ pathway, which causes retinal detachment. Microgl-Exos release IL-1 $\beta$, IFN- $\gamma$ and MCP-2, resulting in a significant inflammatory reaction. Pericyte-Exos carry cPWWPA, which aggravates retinal vascular dysfunction. DR, diabetic retinopathy; ROS, reactive oxygen species; BM-MSCs-Exo, exosomes derived from bone marrow mesenchymal stem cells; TLR, Toll-like receptor; NO, nitric oxide; PRP, platelet plasma; ET-1, endothelin 1; PGI2, prostaglandin 2; Sexos, serum-derived exosomes; Microgl, microglial; MCP-2, macrophage chemoattractant protein 2; cPWWPA, proline-tryptophan-tryptophan-proline domain-containing protein 2A; SIRT1, sirtuin 1; Arg 1, arginase 1; ICAM-1, intercellular adhesion molecule 1; VCAM-1, vascular cell adhesion molecule 1.

provides a schematic of the mechanisms of the involvement of exosomes in the pathogenesis of DR. A previous study (50) has shown that the arginase 1 contained in the Sexos of patients with DR inhibits nitric oxide (NO), endothelin 1 and prostacyclin but increases prostaglandin 2 after being taken up by endothelial cells to cause vascular endothelial damage in DR. In addition, miR-15a carried by Sexos inhibits PKB3/Akt3 to activate ROS, which destroy retinal cells in patients, including pigment epithelial cells, Müller cells and photoreceptor cells, representing a pathogenic mechanism of DR (51). Zhang et al (52) indicated that exosomes derived from platelet plasma target TLR4, which activates the NF- $\mathrm{B} / \mathrm{p} 65$ pathway, causing damage to human retinal endothelial cells, and they determined that these exosomes upregulate the expression of retinal intercellular adhesion molecule 1 and vascular cell adhesion molecule 1 , inducing retinal degradation. Experiments in rats have indicated that miR-29 carried by BM-MSCs-Exo inhibited the Smad2/TGF- $\beta$ pathway, which caused proliferative retinopathy and further detachment of the retina (53). Microglia are immune cells of the retina that are closely related to the progression of DR. Exosomes derived from microglia release interleukin- $1 \beta$ (IL-1 $\beta$ ), IL-3, interferon- $\gamma$ and macrophage chemoattractant protein 2 , resulting in a significant inflammatory reaction in patients (54). In addition, exosomes derived from peripheral cells carry proline-tryptophan-tryptophan-proline domain-containing protein $2 \mathrm{~A}$ and act on angiopoietin 1 , occludin, and sirtuin1, which regulates endothelial cells and aggravates retinal vascular dysfunction in rats (55). The abovementioned studies demonstrated that exosomes affect and participate in the pathophysiological processes of DR via various mechanisms.

Diabetic cardiomyopathy (DCM). DCM is caused by extensive necrosis of the myocardium due to cardiac microvascular disease and myocardial metabolic disorders (56). The prevalence of myocardial damage caused by high glucose in the population is increasing and the progression of this damage is becoming increasingly more rapid. The pathogenesis of DCM is primarily related to metabolic disorders, myocardial fibrosis, myocardial cell apoptosis and microvascular disease (57). Fig. 3 provides a schematic of the mechanisms of the involvement of exosomes in the pathogenesis of DCM. In recent years, studies have indicated that exosomes are closely related to the pathogenesis of DCM. Cardiac fibrosis is the primary cause of myocardial dysfunction in patients with diabetes. The transdifferentiation of fibroblasts into myofibroblasts is key to the process of cardiac fibrosis (58). Experiments in rats suggested that BM-MSCs-Exo carry molecular targeting proteases, such as plasmin, MMP-2 and MMP-9, to activate the TGF- $\beta 1 / \mathrm{Smad} 2 / 3$ signaling pathway to promote cardiac muscle fibrosis, causing myocardial damage (59). Furthermore, miR-204 carried by exosomes derived from islet cells inhibits the expression of insulin receptor substrate 1 in rats, causing insulin resistance. In addition to aggravating myocardial energy mechanism disorders, miR-204 also directly damages the structure and function of the left ventricle (60). In rats, it was demonstrated that ADSC-Exos activate the key promoters of the cardiomyocyte apoptosis pathway, caspase12 and C/EBP-homologous protein, 


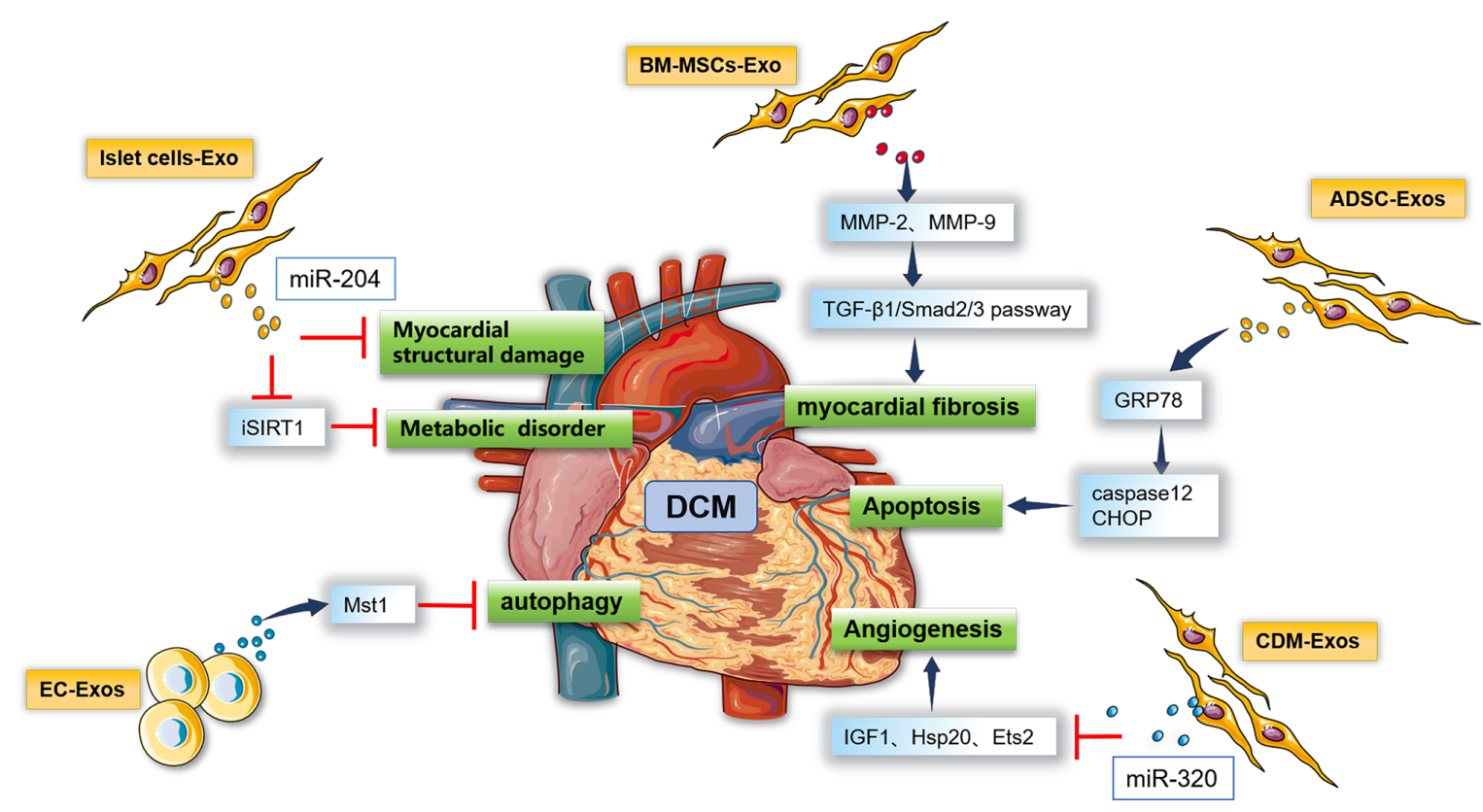

Figure 3. Role of exosomes in the pathogenesis of DCM. BM-MSCs-Exo carry molecular targeting proteases, such as plasmin, MMP-2 and MMP-9, which activate the TGF- $\beta 1 / \mathrm{Smad} 2 / 3$ signaling pathway, thereby promoting cardiac muscle fibrosis. miR-204 carried by islet cells-Exo inhibits the expression of iSIRT1, aggravating myocardial energy mechanism disorders. ADSC-Exos activate the key promoters of the cardiomyocyte apoptosis pathway, caspase12 and CHOP, by targeting GRP78, inducing cardiomyocyte apoptosis. miR-320 carried by CDM-Exos inhibits IGF1, Hsp20 and Ets2, inhibiting cardiac endothelial cell migration and blood vessel formation. EC-Exos release Mst1, inducing cardiomyocyte apoptosis. DCM, diabetic cardiomyopathy; BM-MSCs-Exo, exosomes derived from bone marrow mesenchymal stem cells; ADSC, adipose-derived stem cells; iSIRT1, insulin receptor substrate 1; miR, microRNA; IGF, insulin-like growth factor; Hsp, heat shock protein; CHOP, C/EBP-homologous protein; CRP78, 78-kD glucose-regulated protein; CDM, cardiomyocyte; Ets2, E26 transformation-specific proto-oncogene 2; Mst1, mammalian ste20-like kinase-1; EC, endothelial cell.

by targeting $78-\mathrm{kD}$ glucose-regulated protein and mediating the endoplasmic reticulum response. ADSC-Exos induce cell apoptosis and eventually lead to DCM (61). Observations in rats have also indicated that miR-320 carried by cardiomyocyte exosomes inhibits target gene receptors such as insulin-like growth factor 1 (IGF1), heat shock protein 20 and E26 transformation-specific proto-oncogene 2. The inhibition of cardiac endothelial cell migration and blood vessel formation may be one of the mechanisms of microangiogenesis in DCM (62). Exosomes derived from endothelial cells increase the content of mammalian ste20-like kinase-1 (Mst1), a key protein in the Hippo pathway that regulates organ size, apoptosis and autophagy and enhance the binding of Mst1 and death domain-associated protein to disrupt GLUT4 membrane translocation, thereby aggravating insulin resistance. Furthermore, Mst1 inhibits autophagy of cardiomyocytes, enhances cell apoptosis and promotes the formation of DCM in rats (63). Although exosomes have important roles in the mechanisms of DCM, the evidence available is limited to animal experiments (64) and corresponding clinical studies are lacking. The diabetes-mediated regulation of exosomes in the cardiovascular system is currently under further investigation, which may be a promising treatment approach that will benefit numerous clinical patients.

Diabetic peripheral neuropathy (DPN). DPN may affect any part of the nervous system and includes central nervous system complications, peripheral neuropathy and autonomic neuropathy (65). The pathogenesis of DPN is complex and is primarily related to inflammation under high-glucose conditions. The activation of factors is related to intracranial microvascular disease (66). Fig. 4 provides a schematic of the mechanisms of the involvement of exosomes in the pathogenesis of DPN. It has been indicated that certain exosomes are inflammatory markers of DPN. The miR-21-5p carried by exosomes originating from dorsal root ganglia neurons increases TNF- $\alpha$, IL- 6 and other inflammatory factors, which induces the polarization of macrophages to the proinflammatory M1 phenotype, accelerating the occurrence and development of DPN in rats (67). miR-146a carried by BM-MSCs-Exo also increases TNF- $\alpha$ and mediates the inflammatory reaction (68). Jia et al (69) report that the expression of miR-28, -31a and -130a in Schwann cells-derived exosome is increased by the action of target proteins in axons, such as DNA methyltransferase 3A, Numb protein, synaptosome protein 25 and growth-related protein 43 , which causes rat axon ischemia, hypoxia and abnormal metabolism, inhibits axon growth and aggravates DPN damage. BM-MSCs-Exo carry let-7a, miR-17, miR-23 and other molecules that inhibit the TLR4/NF- $\mathrm{KB}$ signaling pathway, leading to a decrease in neurotrophic factors and ultimately participating in the pathogenesis of DPN in mice (70). Part of the reason for the damage to the nervous system is the disturbance of nervous system homeostasis caused by oxidative stress and increased apoptosis (71). Based on this observation, studies on nerve cells have indicated that exosomes derived from astrocytes activate ROS and target the Ras/adenosine monophosphate-activated protein kinase signaling pathway, which causes mitochondrial dysfunction and apoptosis, ultimately leading to neuronal damage (72). The above mechanistic study suggests the potential of using exosomes in treating DPN. 


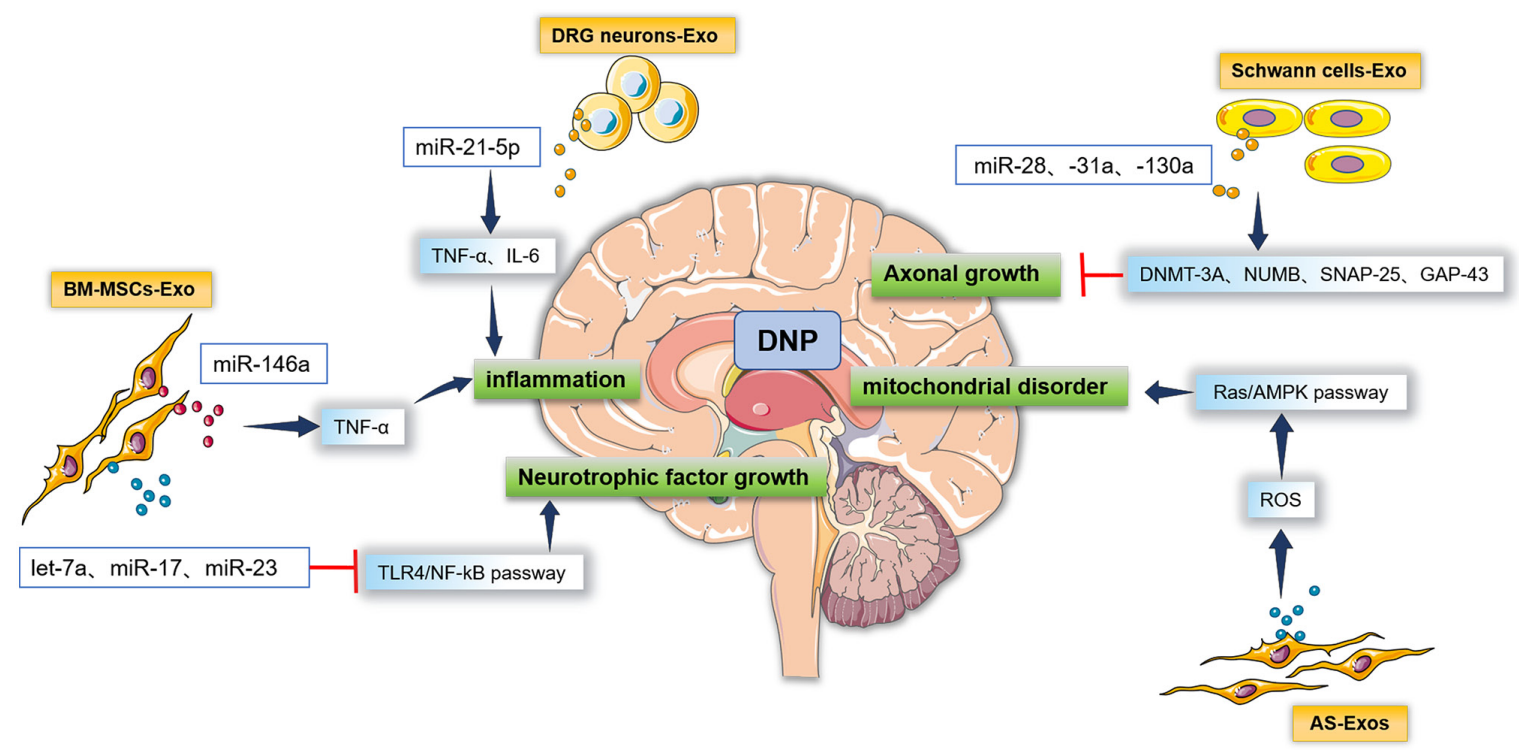

Figure 4. Exosomes are involved in the disease progression of DPN. miR-21-5p carried by DRG neurons-Exo increases TNF- $\alpha$, IL-6 and other inflammatory factors, inducing inflammatory reactions. miR-146a carried by BM-MSCs-Exo also increases TNF- $\alpha$, mediating the inflammatory reaction. The increases in miR-28, -31a and -130a in Schwann cells-Exo target DNMT-3A, NUMB, SNAP-25 and GAP-43, which causes axon ischemia, hypoxia and abnormal metabolism and inhibits axon growth. BM-MSCs-Exo carry let-7a, miR-17, miR-23 and other molecules that target the TLR4/NF- $\mathrm{B}$ s signaling pathway, which leads to a decrease in neurotrophic factors. Under high glucose conditions, part of the reason for the damage to the nervous system is the disturbance of nervous system homeostasis caused by oxidative stress and increased apoptosis. AS-Exos activate ROS and target the Ras/AMPK signaling pathway, causing mitochondrial dysfunction and apoptosis. DPN, diabetic peripheral neuropathy; BM-MSCs-Exo, exosomes derived from bone marrow mesenchymal stem cells; ROS, reactive oxygen species; miR, microRNA; DRG, dorsal root ganglia; DNMT-3A, DNA methyltransferase 3A; SNAP-25, synaptosome protein 25; GRP-43, growth-associated protein 43; AS, astrocytes; AMPK, adenosine monophosphate-activated protein kinase.

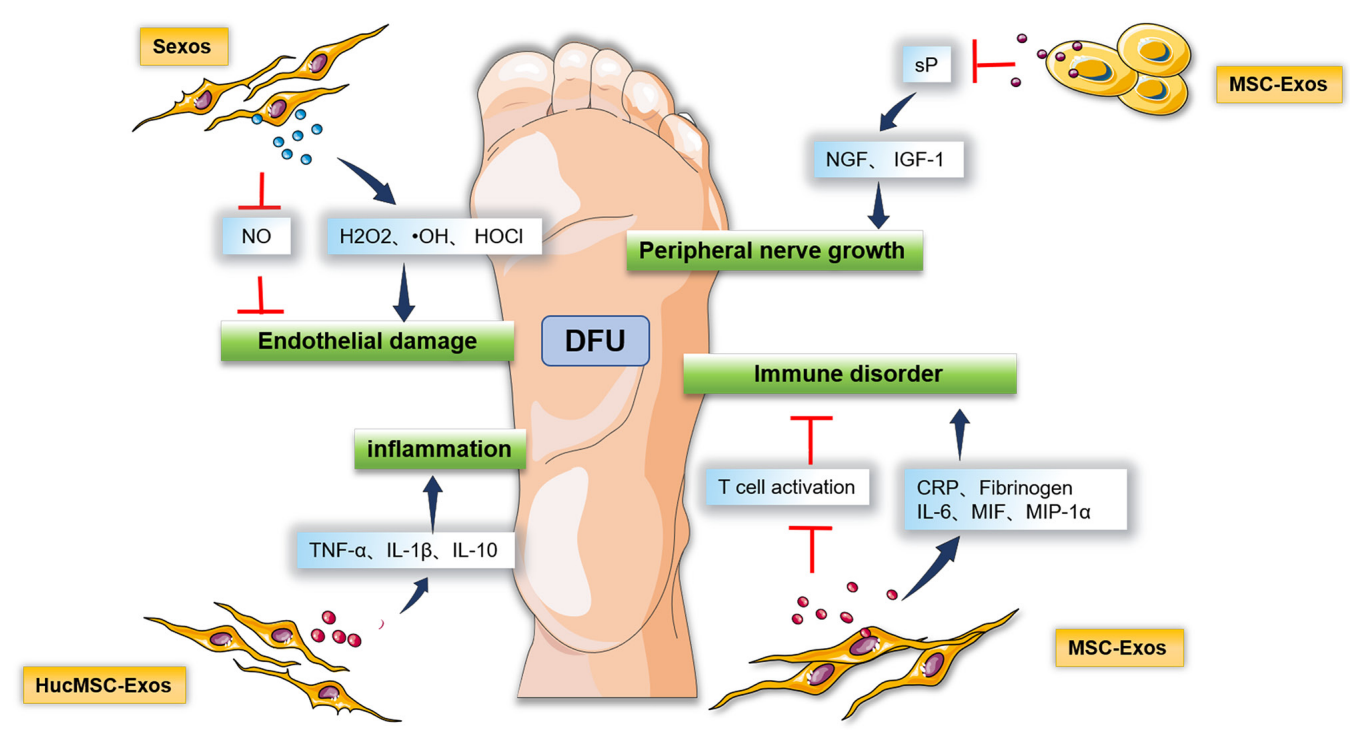

Figure 5. Exosomes are involved in the pathogenesis of DFU. HucMSC-Exos increase TNF- $\alpha$, IL-1 $\beta$ and IL-10, leading to pathological manifestations. Sexos cause the accumulation of $\mathrm{H}_{2} \mathrm{O}_{2}, \bullet \mathrm{OH}$ and $\mathrm{HOCl}$ as well as the reduction of $\mathrm{NO}$, causing damage to the vascular endothelium. MSC-Exos increase CRP, fibrinogen, IL-6, MIF and MIP-1a but decrease the expression and secretion of activated T cells. The molecules carried by MSC-Exos inhibit the production of $\mathrm{sP}$, leading to peripheral nerve regeneration disorders. DFU, diabetic foot ulcer; NO, nitric oxide; CRP, C-reactive protein; HucMSC-Exos, exosomes derived from human umbilical cord mesenchymal stem cells; Sexos, serum-derived exosomes; MIF, macrophage migration inhibitory factor; MIP-1 $\alpha$, macrophage inflammatory protein-1 $\alpha$; sP, substance $\mathrm{P}$.

Diabetic foot ulcer (DFU). DFU refers to foot ulcers, infections and/or deep tissue destruction associated with abnormalities in the distal nerves of the lower extremities and varying degrees of peripheral vascular disease (73). DFU is one of the common complications of diabetes and a chronic disease caused by multiple factors related to skin susceptibility, vascular disease, neuropathy and immune factors under high-glucose conditions (74). In recent years, it has been indicated that exosomes participate in the occurrence of DFU through a variety of mechanisms (75) (Fig. 5). For instance, HucMSC-Exos increase inflammatory factors, such as TNF- $\alpha$, IL- $1 \beta$ and IL-10, leading to pathological manifestations in mice, such as poor granulation 
formation, tissue fragility, delayed epithelialization, tissue rupture and necrosis (76). In addition, exosomes derived from the Sexos of diabetic patients induce the accumulation of hydrogen peroxide, hydroxyl radicals and hypochlorous acid, as well as the reduction of endothelial relaxing factors, such as NO, causing damage to the vascular endothelium, which slows down the repair of diabetic wounds in mice (77). MSC-Exos significantly increase C-reactive protein, fibrinogen, IL-6, macrophage migration inhibitory factor and macrophage inflammatory protein- $1 \alpha$ but decrease the expression and secretion of activated $\mathrm{T}$ cells, which participate in the pathogenesis of DFU in rats (78). The sensory neuropeptide substance P (sP) secreted by nerve endings is a vasoactive substance (79) that mediates nerve regulation in wound repair. The delayed healing of diabetic wounds is closely related to the lack of local sP secretion (80). The molecules carried by MSC-Exos induce the destruction of ulcers under high-glucose conditions and their mechanism of action is to inhibit $\mathrm{SP}$ production. The expression of neurotrophic factors, such as nerve growth factor and IGF-1, decreases, which leads to peripheral nerve regeneration disorders, thereby inhibiting the healing of ulcer wounds in rats (81). Thus, diagnosis and treatment plans may be formulated based on the pathogenesis of DFU resulting from exosomes.

\section{Conclusion}

Due to the complexity of the clinical manifestations underlying different types of diabetes complications, it is challenging to identify them in time by using current laboratory methods (82). However, physiological and pathological changes may be monitored by analyzing the contents of lipids, proteins, nucleic acids and exosomes. In addition, exosomes are simple, cheap and easy to obtain and may meet the demand for novel biological markers to diagnose diabetes in the future (18). In addition, as a natural endogenous carrier of drugs, exosomes have unique advantages: They have good immunocompatibility and low immunogenicity and are able to avoid the rapid clearance of mononuclear macrophages (83). Exosomes may also deliver drugs to specific tissues or organs (84). Therefore, exosomes may overcome the difficulties faced by the current encapsulation of nucleic acid drugs based on miRNAs, which will make it possible for nucleic acid drugs to be used widely in treating diabetic complications in the future (85). However, the pathogenesis of diabetic complications caused by exosomes from different sources has remained to be fully elucidated and more research is required to identify the mechanisms of exosome regulation as a therapeutic target (16). Research focused on exosomes as the entry point will provide novel ideas and methods for the prevention and treatment of diabetic complications in the future and is expected to become a novel strategy for curing diabetic complications.

\section{Acknowledgements}

Not applicable.

\section{Funding}

This study was supported by grants from the National Natural Science Foundation of China (grant nos. 81802446 and 82173371), the Tai Shan Young Scholar Foundation of Shandong Province (grant no. tsqn201909192), the Shandong Provincial Natural Science Foundation (grant nos.ZR2019BH050,ZR2020YQ59 and ZR202103020202) and the Project of Medicine Health and Technology Development Plan of Shandong Province (grant nos. 202003031182 and 202003031183).

\section{Availability of data and materials}

Data sharing is not applicable to this article, as no datasets were generated or analyzed during the current study.

\section{Authors' contributions}

BZ and QB conceived the study. SS performed the literature search. XuL was involved in the writing of the manuscript. DJ and XiL reviewed and revised the article. All the authors read and approved the final version of the manuscript. Data authentication is not applicable.

\section{Ethics approval and consent to participate}

Not applicable.

\section{Patient consent for publication}

Not applicable.

\section{Competing interests}

The authors declare that they have no competing interests.

\section{References}

1. Saeedi P,Petersohn I, Salpea P, Malanda B, Karuranga S, Unwin N, Colagiuri S, Guariguata L, Motala AA, Ogurtsova K, et al: Global and regional diabetes prevalence estimates for 2019 and projections for 2030 and 2045: Results from the international diabetes federation diabetes atlas, 9th edition. Diabetes Res Clin Pract 157: 107843, 2019.

2. Thomas CC and Philipson LH: Update on diabetes classification. Med Clin North Am 99: 1-16, 2015.

3. Müller G: Microvesicles/exosomes as potential novel biomarkers of metabolic diseases. Diabetes Metab Syndr Obes 5: 247-282, 2012.

4. Ying W, Riopel M, Bandyopadhyay G, Dong Y, Birmingham A, Seo JB, Ofrecio JM, Wollam J, Hernandez-Carretero A, $\mathrm{Fu} \mathrm{W}$, et al: Adipose tissue macrophage-derived exosomal miRNAs can modulate in vivo and in vitro insulin sensitivity. Cell 171: 372-384.e12, 2017.

5. Lin J, Li J, Huang B, Liu J, Chen X, Chen XM, Xu YM, Huang LF and Wang XZ: Exosomes: Novel biomarkers for clinical diagnosis. ScientificWorldJournal 2015: 657086, 2015.

6. Kalluri R and LeBleu VS: The biology, function, and biomedical applications of exosomes. Science 367: eaau6977, 2020.

7. Théry C, Zitvogel L and Amigorena S: Exosomes: Composition, biogenesis and function. Nat Rev Immunol 2: 569-579, 2002.

8. Doyle LM and Wang MZ: Overview of extracellular vesicles, their origin, composition, purpose, and methods for exosome isolation and analysis. Cells 8: 727, 2019.

9. Pegtel DM and Gould SJ: Exosomes. Annu Rev Biochem 88: 487-514, 2019.

10. Zhang J, Li S, Li L, Li M, Guo C, Yao J and Mi S: Exosome and exosomal microRNA: Trafficking, sorting, and function. Genomics Proteomics Bioinformatics 13: 17-24, 2015.

11. Lebovitz HE: Etiology and pathogenesis of diabetes mellitus. Pediatr Clin North Am 31: 521-530, 1984. 
12. Kaul K, Tarr JM, Ahmad SI, Kohner EM and Chibber R: Introduction to diabetes mellitus. Adv Exp Med Biol 771: 1-11, 2012.

13. Forbes JM and Cooper ME: Mechanisms of diabetic complications. Physiol Rev 93: 137-188, 2013.

14. Jin J, Shi Y, Gong J, Zhao L, Li Y, He Q and Huang H: Exosome secreted from adipose-derived stem cells attenuates diabetic nephropathy by promoting autophagy flux and inhibiting apoptosis in podocyte. Stem Cell Res Ther 10: 95, 2019.

15. Chang W and Wang J: Exosomes and their noncoding RNA cargo are emerging as new modulators for diabetes mellitus. Cells 8: 853, 2019.

16. Castaño C, Novials A and Párrizas M: Exosomes and diabetes. Diabetes Metab Res Rev 35: e3107, 2019.

17. Kakleas K, Soldatou A, Karachaliou F and Karavanaki K: Associated autoimmune diseases in children and adolescents with type 1 diabetes mellitus (T1DM). Autoimmun Rev 14: 781-797, 2015.

18. Cianciaruso C, Phelps EA, Pasquier M, Hamelin R, Demurtas D, Alibashe Ahmed M, Piemonti L, Hirosue S, Swartz MA, De Palma M, et al: Primary human and rat $\beta$-cells release the intracellular autoantigens GAD65, IA-2, and proinsulin in exosomes together with cytokine-induced enhancers of immunity. Diabetes 66: 460-473, 2017

19. Rahman MJ, Regn D, Bashratyan R and Dai YD: Exosomes released by islet-derived mesenchymal stem cells trigger autoimmune responses in NOD mice. Diabetes 63: 1008-1020, 2014

20. Garcia-Contreras M, Brooks RW, Boccuzzi L, Robbins PD and Ricordi C: Exosomes as biomarkers and therapeutic tools for type 1 diabetes mellitus. Eur Rev Med Pharmacol Sci 21 : 2940-2956, 2017.

21. Tsukita S, Yamada T, Takahashi K, Munakata Y, Hosaka S, Takahashi H, Gao J, Shirai Y, Kodama S, Asai Y, et al: MicroRNAs $106 \mathrm{~b}$ and 222 improve hyperglycemia in a mouse model of insulin-deficient diabetes via pancreatic $\beta$-cell proliferation. EBioMedicine 15: 163-172, 2017.

22. Malone JI and Hansen BC: Does obesity cause type 2 diabetes mellitus (T2DM)? Or is it the opposite? Pediatr Diabetes 20: 5-9, 2019.

23. Heydemann A: An overview of murine high fat diet as a model for type 2 diabetes mellitus. J Diabetes Res 2016: 2902351, 2016.

24. Zhao H, Shang Q, Pan Z, Bai Y, Li Z, Zhang H, Zhang Q, Guo C, Zhang L and Wang Q: Exosomes from adipose-derived stem cells attenuate adipose inflammation and obesity through polarizing $\mathrm{m} 2$ macrophages and beiging in white adipose tissue. Diabetes 67: 235-247, 2018

25. Castaño C,Kalko S, Novials A and Párrizas M: Obesity-associated exosomal miRNAs modulate glucose and lipid metabolism in mice. Proc Natl Acad Sci USA 115: 12158-12163, 2018

26. Brown AE and Walker M: Genetics of insulin resistance and the metabolic syndrome. Curr Cardiol Rep 18: 75, 2016.

27. Sun Y, Shi H, Yin S, Ji C, Zhang X, Zhang B, Wu P, Shi Y, Mao F, Yan Y, et al: Human mesenchymal stem cell derived exosomes alleviate type 2 diabetes mellitus by reversing peripheral insulin resistance and relieving $\beta$-cell destruction. ACS Nano 12 : 7613-7628, 2018

28. Cole JB and Florez JC: Genetics of diabetes mellitus and diabetes complications. Nat Rev Nephrol 16: 377-390, 2020.

29. Barrett EJ, Liu Z, Khamaisi M, King GL, Klein R, Klein BEK, Hughes TM, Craft S, Freedman BI, Bowden DW, et al: Diabetic microvascular disease: An endocrine society scientific statement. J Clin Endocrinol Metab 102: 4343-4410, 2017.

30. Shen B, Liu J, Zhang F, Wang Y, Qin Y, Zhou Z, Qiu J and Fan Y: CCR2 positive exosome released by mesenchymal stem cells suppresses macrophage functions and alleviates ischemia/reperfusion-induced renal injury. Stem Cells Int 2016: 1240301, 2016.

31. Liang X,Zhang L, Wang S, Han Q and Zhao RC: Exosomes secreted by mesenchymal stem cells promote endothelial cell angiogenesis by transferring miR-125a. J Cell Sci 129: 2182-2189, 2016.

32. Chimenti MS, Ballanti E, Triggianese $P$ and Perricone R: Vasculitides and the complement system: A comprehensive review. Clin Rev Allergy Immunol 49: 333-346, 2015.

33. Huang C, Fisher KP, Hammer SS, Navitskaya S, Blanchard GJ and Busik JV: Plasma exosomes contribute to microvascular damage in diabetic retinopathy by activating the classical complement pathway. Diabetes 67: 1639-1649, 2018.

34. Martínez-Castelao A, Navarro-González JF, Górriz JL and de Alvaro F: The concept and the epidemiology of diabetic nephropathy have changed in recent years. J Clin Med 4: $1207-1216,2015$
35. Zhang L, Li R, Shi W, Liang X, Liu S, Ye Z, Yu C, Chen Y, Zhang B, Wang W, et al: NFAT2 inhibitor ameliorates diabetic nephropathy and podocyte injury in $\mathrm{db} / \mathrm{db}$ mice. Br J Pharmacol 170: 426-439, 2013

36. Ioannou K: Diabetic nephropathy: Is it always there? Assumptions, weaknesses and pitfalls in the diagnosis. Hormones (Athens) 16: 351-361, 2017

37. Sakurai A, Ono H, Ochi A, Matsuura M, Yoshimoto S, Kishi S, Murakami T, Tominaga T, Nagai K, Abe H and Doi T: Involvement of Elf3 on Smad3 activation-dependent injuries in podocytes and excretion of urinary exosome in diabetic nephropathy. PLoS One 14: e0216788, 2019.

38. Abe H, Sakurai A, Ono H, Hayashi S, Yoshimoto S, Ochi A, Ueda S, Nishimura K, Shibata E, Tamaki M, et al: Urinary exosomal mRNA of WT1 as diagnostic and prognostic biomarker for diabetic nephropathy. J Med Invest 65: 208-215, 2018.

39. Kim H, Bae YU, Jeon JS, Noh H, Park HK, Byun DW, Han DC, Ryu S and Kwon SH: The circulating exosomal microRNAs related to albuminuria in patients with diabetic nephropathy. J Transl Med 17: 236, 2019.

40. Calle P and Hotter G: Macrophage phenotype and fibrosis in diabetic nephropathy. Int J Mol Sci 21: 2806, 2020.

41. Wang T, Zhu H, Yang S and Fei X: Let-7a-5p may participate in the pathogenesis of diabetic nephropathy through targeting HMGA2. Mol Med Rep 19: 4229-4237, 2019.

42. Ding Y and Choi ME: Regulation of autophagy by TGF- $\beta$ : Emerging role in kidney fibrosis. Semin Nephrol 34: 62-71, 2014.

43. Tervaert TW, Mooyaart AL, Amann K, Cohen AH, Cook HT, Drachenberg CB, Ferrario F, Fogo AB, Haas M, de Heer E, et al: Pathologic classification of diabetic nephropathy. J Am Soc Nephrol 21: 556-563, 2010.

44. Jia Y, Zheng Z, Xue M, Zhang S, Hu F, Li Y, Yang Y, Zou M, Li S, Wang L, et al: Extracellular vesicles from albumin-induced tubular epithelial cells promote the M1 macrophage phenotype by targeting klotho. Mol Ther 27: 1452-1466, 2019.

45. Ding Y and Choi ME: Autophagy in diabetic nephropathy. J Endocrinol 224: R15-R30, 2015.

46. Lu Q, Wang WW, Zhang MZ, Ma ZX, Qiu XR, Shen M and Yin XX: ROS induces epithelial-mesenchymal transition via the TGF-31/PI3K/Akt/mTOR pathway in diabetic nephropathy. Exp Ther Med 17: 835-846, 2019.

47. Antonetti DA, Klein R and Gardner TW: Diabetic retinopathy. N Engl J Med 366: 1227-1239, 2012.

48. Mazzeo A, Beltramo E, Lopatina T, Gai C, Trento M and Porta M: Molecular and functional characterization of circulating extracellular vesicles from diabetic patients with and without retinopathy and healthy subjects. Exp Eye Res 176: 69-77, 2018.

49. Heng LZ, Comyn O, Peto T, Tadros C, Ng E, Sivaprasad S and Hykin PG: Diabetic retinopathy: Pathogenesis, clinical grading, management and future developments. Diabet Med 30: 640-650, 2013.

50. Shosha E, Xu Z, Narayanan SP, Lemtalsi T, Fouda AY, Rojas M, Xing J, Fulton D, Caldwell RW and Caldwell RB: Mechanisms of diabetes-induced endothelial cell senescence: Role of arginase 1. Int J Mol Sci 19: 1215, 2018.

51. Naruse R, Suetsugu M, Terasawa T, Ito K, Hara K, Takebayashi K, Morita K, Aso Y and Inukai T: Oxidative stress and antioxidative potency are closely associated with diabetic retinopathy and nephropathy in patients with type 2 diabetes. Saudi Med J 34: 135-141, 2013.

52. Zhang W, Dong X, Wang T and Kong Y: Exosomes derived from platelet-rich plasma mediate hyperglycemia-induced retinal endothelial injury via targeting the TLR4 signaling pathway. Exp Eye Res 189: 107813, 2019.

53. Shao L, Zhang Y, Lan B, Wang J, Zhang Z, Zhang L, Xiao P, Meng Q, Geng YJ, Yu XY and Li Y: MiRNA-sequence indicates that mesenchymal stem cells and exosomes have similar mechanism to enhance cardiac repair. Biomed Res Int 2017: 4150705, 2017.

54. Vujosevic S, Micera A, Bini S, Berton M, Esposito G and Midena E: Proteome analysis of retinal glia cells-related inflammatory cytokines in the aqueous humour of diabetic patients. Acta Ophthalmol 94: 56-64, 2016.

55. Liu C, Ge HM, Liu BH, Dong R, Shan K, Chen X, Yao MD, Li XM, Yao J, Zhou RM, et al: Targeting pericyte-endothelial cell crosstalk by circular RNA-cPWWP2A inhibition aggravates diabetes-induced microvascular dysfunction. Proc Natl Acad Sci USA 116: 7455-7464, 2019

56. Dillmann WH: Diabetic cardiomyopathy. Circ Res 124: $1160-1162,2019$ 
57. Bugger $\mathrm{H}$ and Abel ED: Molecular mechanisms of diabetic cardiomyopathy. Diabetologia 57: 660-671, 2014.

58. Jia G, Demarco VG and Sowers JR: Insulin resistance and hyperinsulinaemia in diabetic cardiomyopathy. Nat Rev Endocrinol 12: 144-153, 2016.

59. Liu X, Song X, Lu J, Chen X, Liang E, Liu X, Zhang M, Zhang Y, Du Z and Zhao Y: Neferine inhibits proliferation and collagen synthesis induced by high glucose in cardiac fibroblasts and reduces cardiac fibrosis in diabetic mice. Oncotarget 7 : 61703-61715, 2016.

60. Schenk S, McCurdy CE, Philp A, Chen MZ, Holliday MJ, Bandyopadhyay GK, Osborn O, Baar K and Olefsky JM: Sirt1 enhances skeletal muscle insulin sensitivity in mice during caloric restriction. J Clin Invest 121: 4281-4288, 2011.

61. Tao S, Chen L, Song J, Zhu N, Song X, Shi R, Ge G and Zhang Y: Tanshinone IIA ameliorates diabetic cardiomyopathy by inhibiting Grp78 and CHOP expression in STZ-induced diabetes rats. Exp Ther Med 18: 729-734, 2019.

62. Wang X, Huang W, Liu G, Cai W, Millard RW, Wang Y, Chang J, Peng T and Fan GC: Cardiomyocytes mediate anti-angiogenesis in type 2 diabetic rats through the exosomal transfer of miR-320 into endothelial cells. J Mol Cell Cardiol 74: 139-150, 2014.

63. Hu J, Wang S, Xiong Z, Cheng Z, Yang Z, Lin J, Wang T, Feng X, Gao E, Wang H and Sun D: Exosomal Mstl transfer from cardiac microvascular endothelial cells to cardiomyocytes deteriorates diabetic cardiomyopathy. Biochim Biophys Acta Mol Basis Dis 1864: 3639-3649, 2018

64. Quinaglia T, Oliveira DC, Matos-Souza JR and Sposito AC: Diabetic cardiomyopathy: Factual or factoid? Rev Assoc Med Bras (1992) 65: 61-69, 2019.

65. Feldman EL, Callaghan BC, Pop-Busui R, Zochodne DW, Wright DE, Bennett DL, Bril V, Russell JW and Viswanathan V: Diabetic neuropathy. Nat Rev Dis Primers 5: 41, 2019.

66. Ma J, Yu H, Liu J, Chen Y, Wang Q and Xiang L: Metformin attenuates hyperalgesia and allodynia in rats with painful diabetic neuropathy induced by streptozotocin. Eur J Pharmacol 764: 599-606, 2015.

67. Yin Z, Han Z, Hu T, Zhang S, Ge X, Huang S, Wang L, Yu J, Li W, Wang Y, et al: Neuron-derived exosomes with high miR-21-5p expression promoted polarization of M1 microglia in culture. Brain Behav Immun 83: 270-282, 2020.

68. Feng Y, Chen L, Luo Q, Wu M, Chen Y and Shi X: Involvement of microRNA-146a in diabetic peripheral neuropathy through the regulation of inflammation. Drug Des Devel Ther 12: 171-177, 2018.

69. Jia L, Chopp M, Wang L, Lu X, Szalad A and Zhang ZG: Exosomes derived from high-glucose-stimulated Schwann cells promote development of diabetic peripheral neuropathy. FASEB J 32: fj201800597R, 2018.

70. Alomar SY, Gheit R, Enan ET, El-Bayoumi KS, Shoaeir MZ, Elkazaz AY, Al Thagfan SS, Zaitone SA and El-Sayed RM: Novel mechanism for memantine in attenuating diabetic neuropathic pain in mice via downregulating the spinal HMGB1/TRL4/NF-kB inflammatory axis. Pharmaceuticals (Basel) 14: 307, 2021

71. Chen Q, Zhang D, Wang L, Zhang Y, Chen H, Chen F and He Z: Effect of intermittent high glucose on oxygen-glucose deprivation/refurnish neuronal survival. Zhonghua Wei Zhong Bing Ji Jiu Yi Xue 31: 61-66, 2019 (In Chinese).
72. Bonomelli B, Martegani E and Colombo S: Lack of SNF1 induces localization of active Ras in mitochondria and triggers apoptosis in the yeast saccharomyces cerevisiae. Biochem Biophys Res Commun 523: 130-134, 2020.

73. Boulton AJ: Diabetic neuropathy and foot complications. Handb Clin Neurol 126: 97-107, 2014.

74. Noor S, Zubair M and Ahmad J: Diabetic foot ulcer-a review on pathophysiology, classification and microbial etiology. Diabetes Metab Syndr 9: 192-199, 2015.

75. Li X, Xie X, Lian W, Shi R, Han S, Zhang H, Lu L and Li M: Exosomes from adipose-derived stem cells overexpressing Nrf2 accelerate cutaneous wound healing by promoting vascularization in a diabetic foot ulcer rat model. Exp Mol Med 50: 1-14, 2018.

76. Zhu B, Zhang L, Liang C, Liu B, Pan X, Wang Y, Zhang Y, Zhang Y, Xie W, Yan B, et al: Stem cell-derived exosomes prevent aging-induced cardiac dysfunction through a novel exosome/lncRNA MALAT1/NF- $\kappa \mathrm{B} / \mathrm{TNF}-\alpha$ signaling pathway. Oxid Med Cell Longev 2019: 9739258, 2019.

77. Harrell CR, Jovicic N, Djonov V, Arsenijevic N and Volarevic V: Mesenchymal stem cell-derived exosomes and other extracellular vesicles as new remedies in the therapy of inflammatory diseases. Cells 8: 1605, 2019.

78. Li M, Wang T, Tian H, Wei G, Zhao L and Shi Y: Macrophage-derived exosomes accelerate wound healing through their anti-inflammation effects in a diabetic rat model. Artif Cells Nanomed Biotechnol 47: 3793-3803, 2019.

79. Chen O, Donnelly CR and Ji RR: Regulation of pain by neuro-immune interactions between macrophages and nociceptor sensory neurons. Curr Opin Neurobiol 62: 17-25, 2020. Qing L, Chen H, Tang J and Jia X: Exosomes and their MicroRNA cargo: New players in peripheral nerve regeneration. Neurorehabil Neural Repair 32: 765-776, 2018.

80. Qing L, Chen H, Tang J and Jia X: Exosomes and Their MicroRNA Cargo: New Players in Peripheral Nerve Regeneration. Neurorehabil Neural Repair 32: 765-776, 2018

81. Dalirfardouei R, Jamialahmadi K, Jafarian AH and Mahdipour E: Promising effects of exosomes isolated from menstrual blood-derived mesenchymal stem cell on wound-healing process in diabetic mouse model. J Tissue Eng Regen Med 13: 555-568, 2019.

82. DeFronzo RA, Ferrannini E, Groop L, Henry RR, Herman WH, Holst JJ, Hu FB, Kahn CR, Raz I, Shulman GI, et al: Type 2 diabetes mellitus. Nat Rev Dis Primers 1: 15019, 2015.

83. Cho JA, Yeo DJ, Son HY, Kim HW, Jung DS, Ko JK, Koh JS, Kim YN and Kim CW: Exosomes: A new delivery system for tumor antigens in cancer immunotherapy. Int $\mathrm{J}$ Cancer 114: 613-622, 2005.

84. Rani S, Ryan AE, Griffin MD and Ritter T: Mesenchymal stem cell-derived extracellular vesicles: Toward cell-free therapeutic applications. Mol Ther 23: 812-823, 2015.

85. Zhang Y, Bi J, Huang J, Tang Y, Du S and Li P: Exosome: A review of its classification, isolation techniques, storage, diagnostic and targeted therapy applications. Int J Nanomedicine 15: 6917-6934, 2020 